\title{
Final Report \\ for the 1996 DOE Grant supporting Research at the SLAC/LBNL/LLNL $B$ Factory
}

\author{
D. Judd \\ Department of Physics \\ Prairie View A\&M University \\ Prairie View, TX 77446-0355 \\ D. Wright \\ Particle Physics Division \\ Lawrence Livermore National Laboratory \\ Livermore, CA 94551

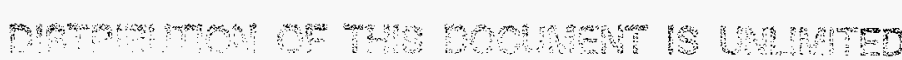 \\ August 8, 1997 \\ Abstract

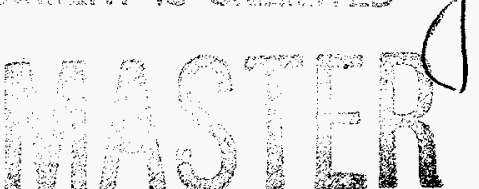

\begin{abstract}
This final report discusses Department of Energy-supported research funded through Lawrence Livermore National Laboratory (LLNL) which was performed as part of a collaboration between LLNL and Prairie View A\&M University to develop part of the $B_{A} B A R$ detector at the SLAC $B$ Factory. This work focuses on the Instrumented Flux Return (IFR) subsystem of $B A B A R$ and involves a full range of detector development activities: computer simulations of detector performance, creation of reconstruction algorithms, and detector hardware R\&D. Lawrence Livermore National Laboratory has a leading role in the IFR subsystem and has established on-site computing and detector facilities to conduct this research. By establishing ties with the existing LLNL Research Collaboration Program and leveraging LLNL resources, the experienced Prairie View group was able to quickly achieve a more prominent role within the $B A B A R$ collaboration and make significant contributions to the detector design. In addition, this work provided the first entry point for Historically Black Colleges and Universities into the $B$ Factory collaboration, and created an opportunity to train a new generation of minority students at the premier electron-positron high energy physics facility in the U.S.
\end{abstract}

\section{DISCLAIMER}

\begin{abstract}
This report was prepared as an account of work sponsored by an agency of the United States Government. Neither the United States Government nor any agency thereof, nor any of their employees, makes any warranty, express or implied, or assumes any legal liability or responsibility for the accuracy, completeness, or usefulness of any information, apparatus, product, or process disclosed, or represents that its use would not infringe privately owned rights. Reference herein to any specific commercial product, process, or service by trade name, trademark, manufacturer, or otherwise does not necessarily constitute or imply its endorsement, recommendation, or favoring by the United States Government or any agency thereof. The views and opinions of authors expressed herein do not necessarily state or reflect those of the United States Government or any agency thereof.
\end{abstract}




\section{DISCLAMMER}

Portions of this document may be illegible in electronic image produets. Images are produced from the best available original document. 


\section{Introduction}

The SLAC/LBNL/LLNL $B$ Factory is an approved High Energy Physics colliding beam facility committed to the observation of $C P$ violation in the neutral $B$ meson system. Since the initial discovery of $C P$ violation in neutral kaons in 1964, the Standard Model mechanism invented to describe it has remained untested. The $B$ Factory is designed to make an unambiguous and over-constrained test of this mechanism by reconstructing $B$ meson decays from electron-positron annihilation. If the results contradict the Standard Model, the multiple independent measurements could indicate the source of the deviation. This may be the first look at new physics beyond the Standard Model.

The $B$ Factory accelerator is now under construction with a scheduled turn on at the beginning of 1999. The $B_{A} B_{A R}$ collaboration has received approval of the Technical Design Report[1] for the collider detector, and has prepared a detailed design of most subdetector components.

\subsection{BABAR}

In order to observe $C P$ violating asymmetries one of the two primary $B$ mesons must be fully reconstructed, the other must be tagged by its weak decay products, and the primary vertex of both must be measured. $B_{A B A R}[1,2]$ is therefore a general purpose, high precision detector consisting of a silicon strip micro-vertex detector, central tracking drift chamber, Čerenkov light based particle identification system, electromagnetic crystal calorimeter (ECAL), magnet coil, and instrumented iron flux return (IFR).

The large superconducting coil, with an inner radius of $1.4 \mathrm{~m}$, generates a magnetic field throughout the central region of the detector. The flux generated by the coil is returned through a hexagonal barrel and endcap configuration of steel plates. The steel plates act as a hadron absorber as well as a path for the magnet field. By placing charged particle detectors, resistive plate chambers (RPC), between the layers of steel, the flux return provides muon and hadronic shower detection capabilities. Section 3.3 contains a detailed description of the IFR system, its physics capabilities and detector technology.

The high energy physics group at LLNL has leading roles in both the IFR and ECAL subsystems. Livermore personnel hold management positions for physics simulation and project engineering and system management in both subdetectors. Livermore physicists are the main architects of the collaboration wide computer simulation of the detector. Also, Livermore has active detector R\&D programs for the chosen technologies for the IFR (Resistive Plate Chambers) and ECAL (cesium iodide scintillating crystals). Livermore is in charge of developing and constructing the inner cylindrical RPC (which goes inside the superconducting solenoid) for the IFR subsystem. 


\subsection{Prairie View Experience}

Prior to joining $B A B A R$, the HEP group at Prairie View was involved in the preparation, running, and analysis of experiment E771 at Fermilab. This work included Monte Carlo studies of the E771 triggers, the design and programming of a large part of the data acquisition system, and the acquisition and installation of the Fastbus readout system for the Central Chambers. They were responsible during the run for the operation of the data acquisition system. After the run ended, they contributed to the first and second pass offline data analysis of the dimuon trigger data. During this time, they acquired considerable expertise in $B$ physics analysis and $\mathrm{C}++$ object-oriented programming, which is directly applicable to the activities outlined in this proposal. Furthermore, they are familiar with RPC technology, since it was used to form the dimuon trigger in E771.

Prairie View has provided an excellent opportunity for undergraduate physics major students to participate in high energy physics research. Section 3.1 gives some statistics for the university and undergraduate physics program, and lists the physics graduates who have graduated from Prairie View and are enrolled in graduate programs.

Before the Fermilab E771 run, four Prairie View physics majors spent 10 weeks at Fermilab, doing the bulk of the installation work on the Central Chambers and their Fastbus readout. The next summer, one of these students also did some work on porting the E771 offline analysis from VMS to UNIX. After the run ended, two physics majors were able to visit Fermilab during the summer, and another one spent the summer at Prairie View, working as a data aide on the E771 offline analysis.

In September 1994, Prairie View joined the $B_{A} B_{A R}$ Collaboration at SLAC and began establishing ties with the LLNL group. During the summer of 1995, one PVAMU student was able to work at Lawrence Livermore National Laboratory: this was George Aduo and he worked in the Physics and Space Science Section doing work on BABAR IFR design and simulation. Four PVAMU faculty members and researchers also worked at LLNL during the summer of 1995, to supervise the student, to familiarize themselves with the work being done at Livermore, and to work on the BABAR research effort with LLNL: these were Prof. Dennis J. Judd, Prof. David E. Wagoner, Prof. Kwang Paick, and Dr. Mei Gui.

During the summer of 1996, 3 students were supported by this grant at Prairie View: they were Johnson Acheampong, Emmanuel Acheampong, and John Cooney. They worked at the Prairie View campus, which had the $B_{A} B_{A R}$ simulation program installed and running on local high energy physics computers. Their work at Prairie View was in the area of $B A B A R$ simulation software with the assistance and supervision of Dr. Margaret Haire and Lon Turnbull. This work also contributed to the preparation needed for the production of Monte Carlo events for the Mock Data Challenge for BABAR. In addition this grant also supported one month of summer research work performed on the BABAR IFR subsystem by Prof. Dennis J. Judd, Prof. David E. Wagoner, and Prof. Kwang Paick. 


\section{Supported Activities}

There are two general categories of tasks involved in the IFR detector program: computation and detector $\mathrm{R} \& \mathrm{D}$. Within the realm of computation we identified three phases of the project that are well matched to the the experience of the Prairie View group that also leverage LLNL capabilities:

Phase I: Detector simulation enhancement and analysis.

Performance studies of the IFR are based on a Monte Carlo simulation, called BBSIM, of the complete detector. BBSIM is a GEANT 3.21 program written primarily in FORTRAN, but with some parts written in C++. Development of BBSIM is being done by many people at many different institutions. Code responsibility is presently divided up by detector component, with different groups being responsible for the Electromagnetic Calorimeter, the Drift Chambers, and so forth. The Prairie View group, together with Livermore and Napoli/Genova, is responsible for the Instrumented Flux Return (IFR). Simulation of the IFR component of the detector has a very high priority, since the IFR will, and must, be the first part of the detector built. It is scheduled to be ready by June of 1997, about a year before other components come on line. During the wait for other detector components, the IFR is expected to be tested with cosmic ray muon triggers. The IFR simulation work is gradually migrating toward reconstruction code, which requires much more communication with other detector components, and toward tracking code, which involves the complete interdependence of the various detector subsystems. Prairie View is beginning to participate more intensively in studying, producing, and testing reconstruction code for the IFR. Specific reconstruction tasks which Prairie View is beginning to investigate include muon tracking in the IFR (especially decoupling the $\phi$ and $z$ position measurements), muon momentum measurement in the IFR, $K_{L}^{0}$ vertex reconstruction, muon track matching, muon/pion separation, and muon particle ID.

Phase II: Object oriented computation.

The current GEANT 3.21 simulation, based on FORTRAN, is expected to be supplanted eventually by the C++ based GEANT4. Concurrently, an object oriented framework, built around $\mathrm{C}++$, is beginning to take shape within the $B_{A} B_{A R}$ computing group. The Livermore group is closely involved with the design of GEANT4 utilities. These are not expected to be ready before the RPCs are built; consequently it will be necessary to do most of the IFR simulation work with the tools available now. LLNL has done a significant amount of work to ensure that $\mathrm{C}++$ and FORTRAN detector code can be written to coexist with GEANT3.21. The Prairie View group has done a large amount of work for the collaboration on using the Hewlett-Packard (HP) C++ compilers, and has also done a large amount of coding in $\mathrm{C}++$ for the IFR simulation. IFR reconstruction software is written entirely in $\mathrm{C}++$. The Prairie View group intends to participate on the GEANT4 simulation effort for $B A B A R$, and on the conversion effort required to convert from the current GEANT3-based BBSIM detailed simulation and fast ASLUND simulation to new combined versions based 
on GEANT4.

Phase III: Massively parallel event simulation.

The Livermore group is a major participant in the laboratory's massively parallel processing initiative and has access to a number of new, massively parallel machines on-site. The goal is to apply this new computing architecture to $B A B A R$ simulation needs in a generic and portable way. Previous forays into parallel processing in high energy physics stopped at the event level. Their approach is to use parallelization at the tracking level, which should reduce event-completion latency and increase turn-around time during the development cycle.

Phase I and II are underway now. Phase III is a long term goal that Prairie View may contribute to after becoming more fully integrated into the current simulation work.

The second major task is detector R\&D. LLNL operates an RPC facility where prototype chambers are produced and tested in a cosmic-ray test stand. Within the $B_{A} B_{A R}$ collaboration, LLNL has responsibility for producing an ultra-thin, cylindrical RPC, developing an assembly and integration scheme for the flat RPCs and finding an environmentally safe, non-flammable RPC operating gas.

One of the goals of this project is to promote the participation of students in high energy physics research. This research work presents opportunities in computing, electronics and detector hardware, providing hands-on experience in all aspects of experimental particle physics.

\section{References}

[1] BABAR Technical Design Report, BABAR Collaboration, SLAC-R-95-457, March 1995.

[2] Letter of Intent for the Study of CP Violation and Heavy Flavor Physics at PEP-II, BABAR Collaboration, SLAC-443, June 1994. 


\section{Appendix}

\subsection{Information about Prairie View A\&M University}

\section{Vital Statistics}

Prairie View, Texas 77446-0355

Student body: 5849 including 969 graduate students

Computer Operations: Kwang Paick

E-mail Address: kwang@hp73.pvamu.edu

409-857-4140

409-857-4144 (Fax)

Network Administrator: None

WAN: Sesquinet@T1 and TAMnet@T1

LAN: Partially complete

Research Activity: High Energy Physics working at SLAC on $B_{A} B_{A R}$ and at Fermilab on E771

PVAMU Physics Graduates Entering Graduate Programs 1992-1996

\begin{tabular}{|lcl|}
\hline PVAMU Graduate & Year Graduated & Graduate Program \\
\hline \hline John Calhoun* & 1992 & University of Iowa \\
\hline Julius Barnes* & 1993 & Rice University \\
\hline Eric Brass* & 1993 & Hampton University \\
\hline Byron Freelon* & 1993 & University of Minnesota \\
\hline Margaret Smith\# & 1993 & University of Michigan \\
\hline Temesgen Habtemariam* & 1994 & University of Minnesota \\
\hline Christopher Johnson & 1994 & Texas A\&M University \\
\hline Eric Johnson & 1994 & University of Minnesota \\
\hline Carlton Watson* & 1994 & University of Iowa \\
\hline Althea Bluiett & 1995 & Michigan State University \\
\hline Titania Dumas & 1995 & University of California, Davis \\
\hline Mohamad Moghadassi & 1995 & Prairie View A\&M University \\
\hline Melynda Price* & 1995 & University of Michigan \\
\hline Juba Watts-Cain & 1995 & University of California, Los Angeles \\
\hline Kevin Storr & 1996 & Florida State University \\
\hline
\end{tabular}

* Passed Qualifying Exam

\# Withdrew from Program 


\subsection{Prairie View A\&M 1996 Status Report}

The high energy physics group at Prairie View A\&M University has been a member of the SLAC BABAR collaboration since September 1994. Our work in the collaboration has so far been focused on computer simulation of the proposed BABAR detector. The simulation program, called BBSIM, is based on the GEANT3.21 program. While GEANT3.21 is written entirely in FORTRAN, much of BBSIM is written in $\mathrm{C}++$. A number of utilities were developed at LLNL to make possible the coexistence of C++ detector code with GEANT3.21. The basic framework of BBSIM was completed, mostly by collaborators at Livermore, before we joined the collaboration, but considerable refinement of the program has been done since then, and further work still needs to be done.

\section{Infrastructure}

In the first few months after joining $B A B A R$, our group was primarily involved with porting BBSIM and its supporting utilities to our HP9000/750 computers. This was a fairly complicated process, since the native $\mathrm{HP} \mathrm{C}++$ compiler was not used at that time to compile BBSIM code. It was necessary to port the GNU C++ compiler, together with GNU gmake utilities, to compile the code. Additional software packages needed were SLAC's rCVS and Software Release Tools, both of which were added to our machines. BBSIM uses a large amount of disk space, so a SCSI disk was added to one of our HPs to accomodate it. We have also been working to try to decrease the szie of BBSIM by, for example, replacing the "dbin" and "FFREAD" utilities with the smaller and more versatile "dbio" utility.

While the basic housekeeping of BBSIM at Prairie View has been completed, there are still updates and changes to the support utilities which need to be done occasionally. A major change to the CVS utility was made after we joined the collaboration, and this change went smoothly. Updates to GNU C++ have not been so successful. Part of the rationale for using GNU $\mathrm{C}++$ was to have a portable compiler for $\mathrm{C}++$ code which would behave the same on all supported platforms. This has proven not to be case with GNU $\mathrm{C}++$, and it remains to be seen if the situation will improve with later releases of the compiler. The Prairie View group has investigated the use of the native HP C++ compilers, studying their problems and possible solutions. Later releases of HP C++ compilers have improved, but continued testing and validation is necessary. Examples of the Prairie View work on BBSIM is given on our following web pages: http://hp73.pvamu.edu/detector.html,

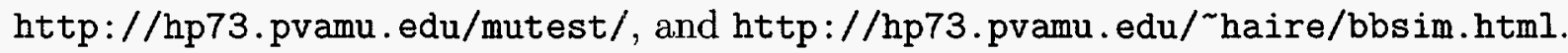

The BABAR collaboration has also moved to the AFS file system. This requires all collaborators to have at least the AFS client software installed and running on their machines.

With the support of this grant, the Prairie View group was able to purchase two new

HP workstations, one of which is resident at PVAMU and the other one at LLNL. All Prairie 
View HP workstations are accessible through the Internet-they are called hp75.pvamu . edu, hp73.pvamu .edu, hp72.pvamu.edu, and llpv1.1lnl.gov.

\section{BBSIM at Prairie View}

Simulation of the IFR component of the $B_{A} B_{A R}$ detector has the highest priority at Prairie View. The IFR is described in detail in the next section. The work we have been doing in the past few months, which is still in progress, is to make a more realistic model of the IFR in the computer simulation. When the TDR was published, the performance of the IFR for muon identification, $\pi / \mu$ separation, and $K_{L}^{0}$ detection was estimated, based on a rather simplistic view of both the IFR steel plates and RPC configuration. Both were taken to be monolithic, with no gaps, and each RPC was represented as a gas gap between graphite plates. It was also assumed that each RPC plane would have a two-dimensional strip readout. A number of simulation changes have been incorporated since then, and a number of hardware design changes have also been necessary.

The first change made by the PVAMU group was to replace the simple RPC plane setup with a more accurate RPC model comprising graphite, bakelite, aluminum, and PVC planes. This work has been completed and incorporated into current BBSIM releases. The second change, being done at Livermore and Prairie View, is to break up the monolithic steel and RPC polygons into real world components, with gaps and supports included. We are now working on the RPCs; Doug Wright at Livermore will incorporate the latest steel design as soon as the Livermore engineers have finished it. Whenever the steel design is refined, a new magnetic field map is required. So far, two iterations of the field map have been done, with the latest coming from Luca Lista (Napoli).

When the TDR was published, we believed that we would be able to put twodimensional RPC strip readouts on every RPC plane. This now cannot be done, due to funding constraints in Italy. It is likely that about $50 \%$ of the planes will have strips in only one dimension. With a TDC on each strip (which is planned), it is possible to convert the output of a one-dimensional readout to a quasi-two-dimensional readout, but there will still be some loss of efficiency. Coding of the reconstruction software, which will take this problem into consideration, has begun in Genova and Napoli. The RPCs themselves, which were assumed to be limited to a maximum length of one meter, have been redesigned with a maximum length of two meters. This will decrease the number of clearance gaps in the barrel. 


\subsection{BABAR Muon and Neutral Hadron Detector}

The iron structure that constitutes the return yoke for the magnetic field is fully instrumented in order to provide muon identification and $K_{L}^{0}$ detection. The Instrumented Flux Return (IFR) consists of a barrel, enclosing the superconducting coil, and two endcaps, and is subdivided into passive steel flux return slabs with active RPC detectors inserted into the intervening gaps between the steel slabs. The total steel thickness required to contain the magnetic flux from the solenoid coil is about $60 \mathrm{~cm}$. This is a good match to the absorber thickness required for a muon filter. Particles reaching the iron will pass through the inner detectors and the coil (a total of 0.9 interaction lengths). To emerge from the iron and strike the outermost chamber particles must pass through an additional 3.6 interaction lengths. This is enough material to reduce the pion punch-through misidentification probability to the percent level in the momentum range between 1 and $3 \mathrm{GeV} / c$, which is comparable to the probability for hadron decay to muons.

Since the measurement of $C P$-violating asymmetries and the experimental program at PEP-II in general are based on exclusive-state reconstruction, the measurement of the hadron energy deposition is not of primary importance. The IFR is not used as a calorimeter for total energy measurement, but as a neutral hadron (primarily $K_{L}^{0}$ ) and muon identifier. Thus, a strip type detector with digital readout is envisioned.

The IFR could also veto cosmic rays at the trigger level by determining the direction (inward or outward) of a track. This could be done by adding a small number of TDCs (one per chamber in a few layers) to measure the time of the first hit in the chambers. Cosmic rays could be well separated from events originating at the interaction point, even with a modest time resolution (in the range of a few nanoseconds).

\section{Design Considerations}

The physics objectives driving the performance required of the IFR detector are: $B$ (and $D$ ) tagging with muons, study of semimuonic decays, reconstruction of $J / \psi \rightarrow \mu^{+} \mu^{-}$, and $K_{L}^{0}$ identification in the $B$ decay products. To reach these goals, the IFR should deliver $\mu-\pi$ separation from $\approx 4 \mathrm{GeV} / c$ down to as far below $1 \mathrm{GeV} / c$ as possible (muons from the charm quark cascade peak at $\approx 500 \mathrm{MeV} / c$ ), with high efficiency and little misidentification; and $K_{L}^{0}$ identification, with directional information, in the energy range of $1-3 \mathrm{GeV}$. The light quark ( $u d s)$ contribution is almost entirely from pion and kaon decay.

The total detector surface and the number of readout channels are the key factors in the cost estimate of the subsystem. Detailed Monte Carlo simulation studies of the full detector with single particle and $\Upsilon(4 S)$ event inputs have addressed the issues of IFR design, focusing on optimizing the iron segmentation. The impact of detector inefficiencies (mostly due to inactive areas) on performance have also been investigated, as well as the merits of 
using double layers. The number of readout channels, determined by the size of the strips and the number of layers to be equipped with bi-dimensional readout, have also been optimized. The benefit of inserting an additional detector layer between the CsI calorimeter and the coil has been investigated, and such a detector (the cylindrical inner RPC) is now being constructed by LLNL.

The optimization process also includes detailed studies of muon identification efficiency $v s$. hadron contamination, as a function of momentum and angle of incidence; $B$ tagging efficiency, in particular for benchmark channels; and $K_{L}^{0}$ identification and angular resolution in $B^{0} \bar{B}^{0}$ events.

\section{Muon Identification}

Since the interaction region is enclosed by the CsI calorimeter and magnet solenoid, muons with momenta below $400 \mathrm{MeV} / c$ do not reach the IFR. Those muons that do reach the IFR must be distinguished from backgrounds resulting from primary charged hadrons (most pions) and secondaries from hadronic showers and decays.

Considering their mass difference and ignoring hadronic interactions, pions and muons differ in their most probable penetration depth (range) in iron by approximately $2-3 \mathrm{~cm}$ for momenta below $1 \mathrm{GeV} / c$. This effect is overshadowed by the high pion-nuclear interaction cross-section. Muons with momenta greater than $1.5 \mathrm{GeV} / c$ completely penetrate the IFR iron.

To exploit the range difference between pions and muons for pion rejection, the momentum of the charged particle must be measured. Furthermore, the charge of the muon must be determined for $B^{0}$ tagging. The central tracking chamber provides this information; its geometrical acceptance is less than that of the flux return, thereby restricting the solid angle for muon identification.

Depending on how much loss of muon efficiency one can tolerate, a range cut reduces the pion misidentification probability to a few percent. Monte Carlo studies have indicated that reducing the iron plate thickness to $2.5 \mathrm{~cm}$ from $0.5 \mathrm{~cm}$ did not significantly improve the pion rejection. Pion background can be further reduced by requiring a high degree of continuity in the pattern of hits in the IFR. Muons tend to give a hit in every layer they go through; pions initiate hadronic showers that can 'skip' detection layers.

Preliminary Monte Carlo studies indicate that muon identification can be done with high efficiency (above 90\%) and low contamination (a few \%) from 500 to $1000 \mathrm{MeV} / \mathrm{c}$ momentum, using a total thickness of $40 \mathrm{~cm}$ of iron divided into plates of approximately $2 \mathrm{~cm}$. The remaining portion of the IFR is divided into thicker plates without seriously compromising performance. Monte Carlo studies have been done to optimize the choice of plate thickness and to verify the impact of segmentation on $K_{L}^{0}$ detection. 


\section{$K_{L}^{0}$ Detection}

The granularity of the IFR detector is also driven by the goal to identify the $K_{L}^{0}$ 's produced in $B$ decays. A sizable fraction of $K_{L}^{0}$ produced in $B^{0} \bar{B}^{0}$ interact in the CsI calorimeter. Preliminary Monte Carlo studies have shown that the fraction of all $K_{L}^{0}$ which reach the IFR varies according to the hadron simulation package used; the experimental data seem to indicate the $K_{L}^{0}$ cross sections on nuclei are overestimated in the GHEISHA package and agree with those used in FLUKA. This is consistent with our findings and encourages us to use FLUKA for these studies.

Some preliminary results from a simulation employing FLUKA indicate that the probability of identifying a $2 \mathrm{GeV} / c K_{L}^{0}$ requiring at least 5 layers hit is about $70 \%$ for iron plates $1 \mathrm{~cm}$ thick, dropping to $\approx 50 \%$ if the plates are $2 \mathrm{~cm}$ thick. Showers produced by photons of the same momentum in the CsI calorimeter do not penetrate past the first 1$2 \mathrm{~cm}$ of iron. Requiring no energy to be deposited in the CsI calorimeter (i.e. selecting those $K_{L}^{0}$ 's that interact directly in the IFR) reduces the identification probability to $20-25 \%$. The angular resolution is typically a few degrees, which is adequate for the kinematic cuts used to reconstruct $B^{0} \rightarrow J / \psi K_{L}^{0}$ final states. A detector layer inserted before the coil may be of real benefit here and is being investigated.

\section{The Active Detectors}

The instrumentation of the flux return cover a surface of $\approx 4000 \mathrm{~m}^{2}$, has a total of $\approx 50,000$ readout channels and is inserted into the gaps between the steel plates. The main requirements for the detector technology are: long term reliability, sturdiness, simplicity of construction and operation, and low cost of the raw materials and readout electronics.

A well-known technique that satisfies these requirements are Resistive Plate Chambers (RPC). RPCs are constructed of two parallel plate electrodes of high electrical resistance (typically bakelite) held apart by insulator spacer buttons and filled with a gas mixture typically consisting of argon, isobutane, and Freon. With DC high voltage applied across the electrodes, a charged particle that crosses the gap will initiate a spark-like discharge which terminates when the electric field collapses due to the depletion of charge on the plates. The spark induces large pulses on external metallic strip-lines which may be read out to determine the initial particle's position.

RPCs have been used successfully in the NADIR experiment at Grenoble, FENICE at Adone (Frascati), E771 at FNAL and WA92 at CERN, and in several cosmic ray detectors. A recent large scale application of this technique is the trigger system for the L3 muon endcaps, consisting of $600 \mathrm{~m}^{2}$ of double gap chambers. This design has an independent high voltage supply to each gap, resulting in more than $99 \%$ efficiency and providing a backup capability in case of failure. This feature seems particularly attractive for a system that is 
difficult to access, such as the IFR. Both sides of the high voltage plane are active gaps, with the readout strips in the middle. A two-dimensional scheme, with two planes of strips running in orthogonal directions, has also been proposed.

After studying a wide variety of materials, LLNL has built several large RPCs that employ a doped ABS plastic sheet as the resistive electrode. Doped ABS plastic, manufactured as a static dispersive packaging material for electronics components, is a superior resistive electrode material because of the strict quality control of its resistivity during manufacturing and its ease of handling (it is shipped in wide rolls).

The main issue with all current types of RPCs is the safety of the gas mixture. The standard RPC gas contains a flammable concentration of isobutane $(\approx 40 \%)$ and a small amount of Freon 13B1, which is a soon to be banned ozone depleting compound. The isobutane must either be replaced by a non-flammable gas or reduced to an acceptable concentration, and a Freon substitute must be found. 


\subsection{Activities of the Theoretical Group}

The experimental HEP group at PVAMU established a theoretical component in July of 1992. Dr. Dan-Di Wu joined and then began to lead the theoretical effort in early August of that year. Over the past five years theoretical physics is beginning to take its place at PVAMU and many contibutions are being made in the area of $B$ physics

Dr. Wu is a strong contributor in the area of $B$-physics and $C P$ violation. He has published more than twenty papers in this field. Several of his papers are frequently cited and are a part of the SLAC/DESY database systems. His 1986 paper on rephasing invariants has been cited more than one hundred times. His recent publications in this field (since coming to PVAMU) include four preprints, with two of the preprints published in Physics Letters B.

The theoretical research at PVAMU is now focused on: 1) the improvement of the accuracy of analysis, especially to disentangle the desired $C P$ violating parameter and the parameters of strong interactions such as the final state phase shift; 2) to test $C P T$ symmetry[6$8]$ in neutral $B$ decays at both symmetric and asymmetric electron-positron $B$ factories or at hadron machines; 3 ) more reliable predictions of branching ratios and $C P$ asymmetries in some experimentally-favored decays of charged and neutral $B$ mesons; improved methods of calculating $B$ mesons to baryon-anti-baryon decay modes; and further studies of the role of QCD-loop-induced (penguin) diagrams in rare $B$ decays and $C P$ violation; 4) joint work (with experimentalists) on event simulation is under consideration, as Dr. Wu has published a paper on an SSC event simulation.

Dr. Wu is also interested in the mass problem and neutrino physics, which are related to $C P$ violation in a more general context. The following is a list of theoretical publications from PVAMU:

\section{List of Recent Theoretical Publications}

1. T. Garavaglia, W. K. Kwong, and D. D. Wu. "Higgs particle detection using jets". Phys. Rev. D 48 (1993) R1899.

2. D. H. Zhang, D. D. Wu, D. Wagoner, and D. Judd. "The calculation of a high order QCD process: $g g$ to $t t g g$ ". Submitted to Comm. Theo. Phys., 1996.

3. Dan-Di Wu and Zhizhong Xing. "On the unitarity triangles of the CKM matrix". Physics Letters B 341 (1995) 386-390.

4. H. Fritzsch, D. D. Wu, and Z. Z. Xing. "On measuring $C P$ violation in neutral $B$-meson decays at the $\Upsilon(4 S)$ Resonance". Phys. Lett. B 328 (1994) 477-502.

5. D. D. Wu. "Small elements in fermion mass matrices and anomalous dipole moments". Phys. Lett. B 374 (1996) 100-110. 
6. D. D. Wu and Y. L. Wu. "Fermion masses and $C P$ violation in $\mathrm{SO}(10)$ models". Modern Physics Letters A11 (1996) 2703-2709.

7. D. D. Wu. "FCNC, $C P$ violation, and impure Majorana neutrinos". e-archive hepph/9612271 and Prairie View A\&M HEP-3-96. Submitted to Physical Review D.

8. D. D. Wu. "A different mechanism to wipe out neutrino oscillations". HEP-3-97. Submitted to Physical Review D.

9. Dan-Di Wu and Zhizhong Xing. "On fine effects in $C P$ violation". To be submitted to Phys. Rev. D.

10. D. D. Wu. "On the test of $C P T$ conservation", 1995.

11. J. Z. Wu, D. D. Wu, Y. Zhou, and X. H. Wu. "Helical wave decomposition in weakly compressible fluid dynamics". Proceedings of Prairie View A\&M First Mechanical Engineering Department Symposium, 1995.

12. J. Z. Wu, D. D. Wu, and Y. Zhou. "Vorticity and strain rate tensor alignment". Proceedings of the Symposium of Engineering and Architecture, 1996.

13. Contributions to conferences: 3-4 presentations per year at American Physical Society Texas meetings, topical conferences and workshops during the past five years. 